\title{
Direct-to-consumer genomic testing: systematic review of the literature on user perspectives
}

\author{
Lesley Goldsmith $^{1}$, Leigh Jackson ${ }^{1}$, Anita O’Connor ${ }^{1}$ and Heather Skirton ${ }^{\star, 1}$
}

Genetic tests have traditionally been offered by health professionals. However, genomic tests have been available direct to the consumer for the last decade, increasingly via the Internet. The aim of this systematic review was to ascertain the evidence concerning use of direct-to-consumer genomic testing from the consumer perspective. Primary research was identified using the search terms 'direct-to-consumer' and 'genomic or genetic' in six bibliographic databases and citation searching of findings. In all, 17 papers were reviewed: 3 qualitative and 14 quantitative. Findings indicate a low level of awareness of direct-to-consumer genomic testing and, because of the hypothetical nature of many studies, little evidence from users of such tests. Although potential users appear to be interested in information about their risks of developing common diseases, concerns were expressed about privacy of genetic risk information and the reliability of genomic tests. Consumers were anxious about the nature of the results. There appeared to be a preference to access genomic tests via a health professional, or to discuss the results and obtain advice from a health professional. Authors of only two papers recruited participants who had used direct-to-consumer tests and samples from the large quantitative studies were not representative of the population. These factors limit the value of the available evidence. However, we conclude that there is public interest in direct-to-consumer genomic tests, and that this is likely to result in an increased workload for a range of health professionals. We also consider that there are educational implications for both consumers and health professionals.

European Journal of Human Genetics (2012) 20, 811-816; doi:10.1038/ejhg.2012.18; published online 15 February 2012

Keywords: direct-to-consumer; genomic testing; genetic testing; health professionals; consumers

\section{INTRODUCTION}

Rapid advances in the field of health genetics have increased the opportunities for genetic testing with a range of applications. ${ }^{1}$ Diagnostic and predictive or presymptomatic tests can enable identification of 'alterations in a single gene or a few genes strongly associated with disease onset' ${ }^{2}$ ( $\mathrm{p} 430$ ). More recently, susceptibility testing based on genome-wide association studies (GWAS) now offers the possibility of approximating an individual's susceptibility to a range of common diseases such as diabetes, ischaemic heart disease and depression. ${ }^{3}$ However, there are concerns that the results of some of these tests may be of limited value, as they are based on limited GWAS that could infer only minimal alterations in risk ${ }^{4}$ and companies may use different genetic markers with varying levels of heritability, thus limiting clinical utility. ${ }^{5}$

Genetic tests with significant predictive value have traditionally been offered in the context of health services, whether private or statefunded, accompanied by pre- and post-test counselling from a health professional, often a genetic counsellor or medical geneticist. ${ }^{6}$ This type of test is now available direct to the consumer (DTC), usually via the Internet (see Table 1), although there have been attempts in the United States (US) to offer such tests in retail pharmacies - these were vetoed by the US Food and Drug Administration. ${ }^{7}$

As in any health-care context, it is important to ensure that the consumers of genomic testing are provided with sufficient information and evidence to facilitate fully informed consent; ${ }^{8}$ this is enormously challenging in the context of DTC tests accessed via the
Internet. It is therefore important to provide health professionals with information to enable them to provide support to any of their patients who choose to access these DTC tests. However, there is little empirical data about how consumers use DTC tests and how they react to the information provided by them. ${ }^{9}$ The aim of this systematic review, therefore, was to identify evidence on the attitudes towards and knowledge and use of DTC tests by members of the general population (potential consumers). This body of evidence will inform the production of guidelines for future consumers of DTC tests, produced as part of the EuroGentest2 project (http://www.eurogentest.org/) and is consistent with the recommendations of the European Society of Human Genetics (ESHG) professional policy committee $(2011)^{10}$ that high quality information should be available to potential customers. This will complement previous guidance published by the ESHG, ${ }^{11}$ which was chiefly aimed at health professionals. The objectives of the review were to: (1) explore the knowledge of and use of DTC genomic tests by the members of the general population, (2) identify the attitudes of members of the general population towards this type of test and (3) identify any risks and benefits of offering genomic tests DTC from the consumer's perspective.

\section{MATERIALS AND METHODS}

Conducting a systematic review is a way of identifying evidence of adequate quality about a specific topic and synthesising it in a way that is rigorous and transparent. ${ }^{12}$ In this study, we used the method described by the Centre for 
Reviews and Dissemination, ${ }^{13}$ which includes a rigorous search strategy, use of inclusion and exclusion criteria and quality appraisal of included papers. The search terms used for this review were 'direct-to-consumer' AND 'genetic or genomic' anywhere in the text of the paper. Papers were restricted to those published between 2001 and 2011, in English. We included primary research papers and systematic reviews. We excluded research relating to DTC marketing of genomic tests, DTC nutrigenomic tests, DTC company strategies or the views and attitudes of health professionals. The databases used were CINAHL, PubMed, Zetoc, PsycINFO, Embase and Medline. From these six sources, a total of 183 papers were identified. These were then read by two authors independently, either as full text $(n=73)$ or abstracts $(n=110)$; this process led to a further reduction in relevant papers, with a total of 16 papers fulfilling the inclusion criteria (see Figure 1). Several papers were identified in more than one database and four did not satisfy the inclusion criteria. A further five relevant papers were added following detailed searching of the references lists of papers already identified and a paper received via a journal alert. The final cohort of papers to be reviewed therefore consisted of 14 quantitative and three qualitative studies.

Table 1 Examples of genomic tests available direct to the consumer (DTC)

\begin{tabular}{ll}
\hline Company & Tests offered \\
\hline $\begin{array}{l}\text { deCODEme (http:// } \\
\text { www.decodeme.com) }\end{array}$ & $\begin{array}{l}47 \text { Conditions including Alzheimer disease, asthma, } \\
\text { breast cancer (not BRCA1/2), heart attack, gallstone, } \\
\text { lung cancer }\end{array}$ \\
$\begin{array}{l}\text { 23andMe (http:// } \\
\text { www.23andme.com) }\end{array}$ & \begin{tabular}{l} 
Carrier testing, pharmacogenomics, susceptibility to \\
\hline
\end{tabular} \\
\hline
\end{tabular}

\section{Quality appraisal}

The quality of the papers was assessed using the Kmet tool for appraising quantitative and qualitative research. ${ }^{14}$ Using this tool, a score of between 0 and 1 was assigned to each paper based on a series of questions related to the type of study. Scores ranged from 0.68 to 0.95 and all papers were therefore considered to be of adequate quality for inclusion. The quality scores are provided in the Supplementary Table as an indicator of the rigour of each study.

\section{Data collection and synthesis}

The papers identified for this systematic review included quantitative and qualitative studies; it was not appropriate therefore to conduct a meta-analysis of the data. The papers were read in depth, themes agreed by three authors and the main features of each were summarised and presented in the Supplementary Table. Further qualitative data were extracted from the findings in the form of basic codes. The codes were scrutinised by two of the authors and grouped into categories. Major themes were identified using thematic analysis ${ }^{15}$ and the findings are presented under these themes.

\section{FINDINGS}

\section{Description of the studies}

Of the 17 papers in the review, 14 reported quantitative studies and three qualitative studies. Twelve were conducted in the United States, one jointly between Canada and the US, with one from the UK and one produced by authors from Belgium and the Netherlands. Two papers focussed on data collected in Australia from the same study. Several of the studies created artificial situations that included the offer of a pseudo direct-to-consumer personalised genomic test to participants. ${ }^{16-21}$ In some studies, participants were questioned about a hypothetical situation rather than one they had actually experienced;

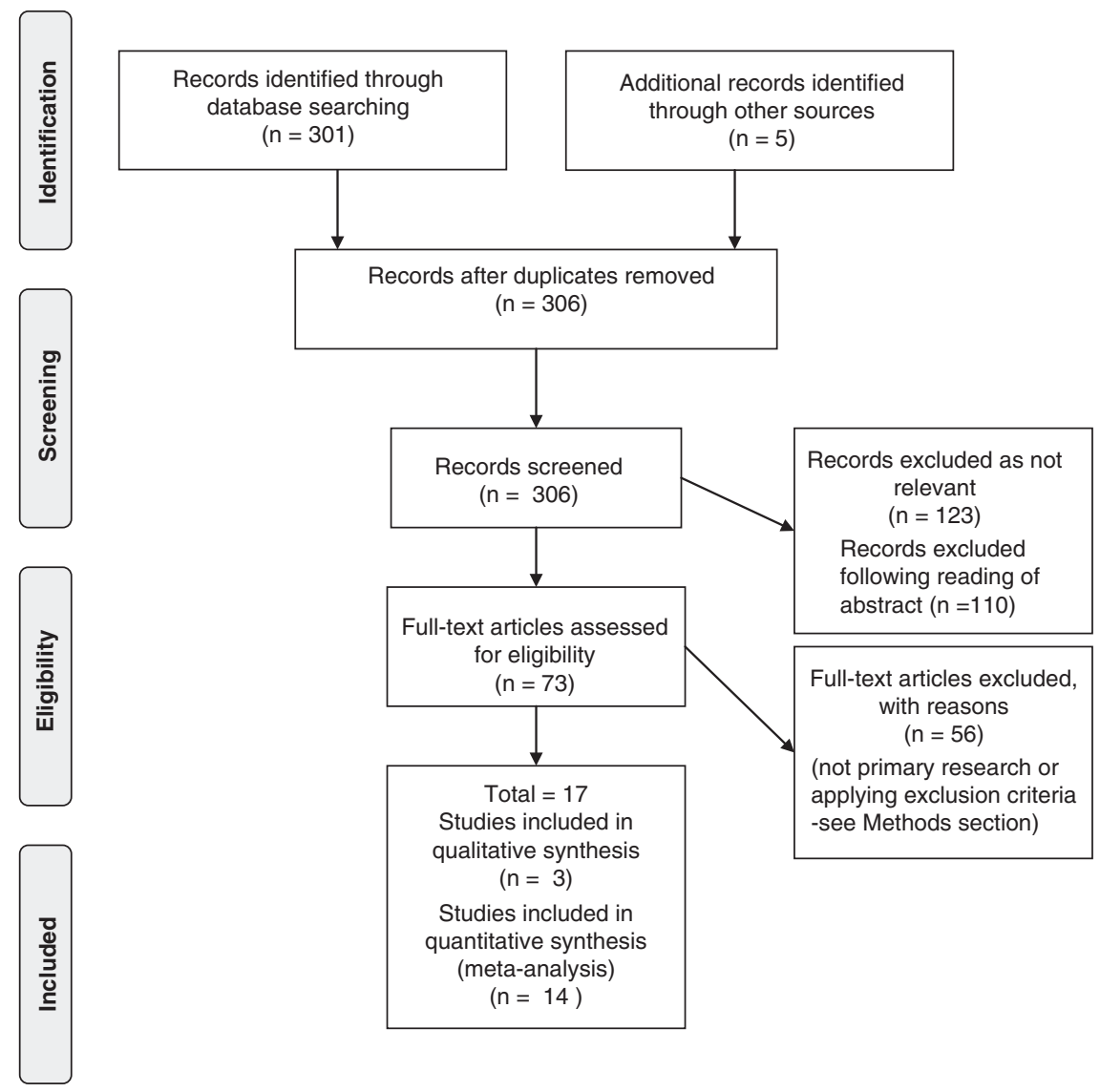

Figure 1 PRISMA 2009 flow diagram. 
these studies investigated participants' intentions to be tested and their decision-making processes rather than their actions ${ }^{22,23}$ or their attitudes and expectations of personal genome testing (PGT). ${ }^{24-27}$ Only two studies involved participants who had experienced DTC tests of their own volition..$^{28,29}$ Some studies were part of large-scale longitudinal projects with large sampling frames - for example, the Scripps Genomic Health Initiative, ${ }^{16,17}$ the Multiplex Initiative ${ }^{19,20}$ and the Coriell Personalized Medicine Collaborative. ${ }^{18}$ Study sample sizes ranged from 84 to 4050 (quantitative) and from 23 to 56 (qualitative). We will now outline the major themes that were identified from the studies.

\section{Reasons for having (or intention to have) a DTC test}

Not surprisingly, the main reason for embarking on a DTC test was to gain detailed genomic information about oneself. Most respondents wanted to learn more about themselves, were curious about their genetic make-up or wanted to learn about individual genetic risk factors. ${ }^{18,22,28}$ Gollust et al $^{18}$ found that over $50 \%$ of participants wanted to ascertain their risk for a particular condition, the most common being heart disease (58\%). In a large quantitative study using the TwinsUK database as a sampling frame, Cherkas et $a^{22}$ found that the most frequent reason given for intention to pursue a test was the adoption of a healthier lifestyle if the result showed a higher than average risk. In all, 53\% stated that a personal genomic test would increase their control over their health and $58 \%$ considered that it would stimulate family discussion about health. ${ }^{22}$ These findings are reinforced by the study by McGowan et al, ${ }^{28}$ in which participants were early users of DTC personal genome scans; participants stated that they would use information gained from the test to take personal responsibility for their future health. Bloss et al ${ }^{16}$ found that over $82 \%$ of participants would want to know their risk of developing any of the common diseases tested, but another study suggested that this level of interest was dependent on cost..$^{22}$

Cherkas et $a^{22}$ also found that $79 \%$ intended to have the test so that their doctor could monitor their health, and $80 \%$ wanted to convey the risk to their children. Su et $a l^{29}$ in a study on the motivations and expectations of people who had actually used DTC tests, collected data from 'blogs' on two sets of websites via a Google search (DTC websites and non-DTC websites) and found that the major theme for motivation on the DTC websites was 'health', whereas themes arising on the blogs of the non-DTC websites were more related to curiosity, fascination, genealogy, contributing to research and recreation. The authors acknowledged that it was difficult to check the validity of blog entries, particularly on DTC company websites where they may have been screened.

\section{Awareness, knowledge and understanding of personal genomic testing}

It appears that the level of awareness and knowledge, and experience of testing in some samples studied was low. Cherkas et al ${ }^{22}$ found that only $13 \%$ of participants (in a UK-based study) had heard of PGT. In contrast, Ortiz et al, ${ }^{30}$ in a study in Puerto Rico, found that a majority $(56 \%)$ were aware of DTC testing although only $4 \%$ had ever used any type of genetic test; awareness was lower in men, single people and smokers, and also in people who were not seekers of information on such health issues as cancer. McGuire et $a^{24}$ stated that only $6 \%$ of participants had used PGT, but on receiving information, $64 \%$ would consider undergoing such a test. Knowledge and understanding of genetics has been found to be a predictor of interest; in the study by McBride et $a l,{ }^{20}$ confidence in understanding genetics predicted whether or not participants logged onto the study website and
McGuire et $a^{24}$ stated that $<50 \%$ of their participants were confident about understanding the risks and benefits of PGT or knew enough to understand the results. Leighton et $a^{\beta 1}$ in a study comparing the understanding of genetic counsellors and members of the general public, found that 'public' respondents were not more likely to correctly interpret DTC results simply because they thought they were easy to understand. They did find, however, that the majority of their 'public' respondents interpreted the results correctly. In their study of early users, McGowan et a ${ }^{28}$ found that despite the concerns expressed by experts in the field, their participants had realistic expectations and were fully aware of the limitations of genomic tests currently being offered direct-to-consumer. Unlike other studies showing that many participants viewed these tests as diagnostic rather than simply expressing a probability - for example, McGuire et al, ${ }^{24}$ and McGowan et $a l^{28}$ found that participants understood the low predictive power if the result was taken alone, and were aware that the results were best viewed in combination with other traditional health risk factors. This finding was supported by Gollust et $a l^{18}$ who found that most people accepted that risk of common disease was multifactorial, however, most also believed that taking part in the Coriell study would provide them with the health-related benefits such as behaviour change. An indication of the low level of understanding by some participants was that $13 \%$ in this study thought that having the genomic test would give them access to gene therapy. Gollust et al $l^{18}$ also considered that people over 55 years were less likely to have misperceptions about personal genomics.

\section{Attitudes and concerns relevant to DTC testing}

One of the main concerns about DTC testing appeared to be that of privacy. In the study by McGuire et al, ${ }^{24} 39 \%$ of those who had not undergone testing and who had no intention to do so, had concerns about privacy; this finding was reinforced by Bloss et al ${ }^{16}$ who found that $36 \%$ had similar concerns. These authors also commented that females, those in health-related occupations, those with poor perceived health, of a younger age, lower income and higher trait anxiety were most likely to express concerns. Wilde et $a l^{27}$ conducted a qualitative study exploring susceptibility testing for depression in a sample of 36 patients selected to be representative of the Australian population as a whole; these participants unanimously rejected the idea of DTC testing, citing the security of the DNA sample and privacy of genetic risk information as two of their main concerns. In a paper reporting quantitative findings from the same study, Wilde et al ${ }^{26}$ stated that interest in accessing genotyping via a doctor was significantly greater than interest in DTC testing, regardless of whether or not the participants had received information.

Another concern expressed by consumers was the nature of the results and how this would affect them. Bloss et al ${ }^{16}$ stated that $13 \%$ were concerned about learning of the disease risk, and 16\% expressed uncertainty about their feelings on hearing the results, while $21 \%$ of participants in the study by McGuire et al ${ }^{24}$ were concerned about 'unwanted information'. Similarly, Gollust et al ${ }^{18}$ found that the most common concern was that the results would cause worry, and that respondents would obtain results they did not want to hear. A final concern was that of the reliability of the test results: concern was expressed by between $16 \%{ }^{12}$ and $21 \%^{20}$ of participants.

\section{Factors influencing the decision to undergo a DTC test}

Some of the larger quantitative studies conducted statistical analyses to identify personal and situational variables that would predict intention of undertaking DTC testing. Several studies found that males were either more likely to undergo tests than females ${ }^{26}$ or that genomic tests 
were viewed less positively by women. ${ }^{16,32}$ This could be explained by the fact that women were significantly more likely to express concerns. ${ }^{16}$ Presentation of risk information appears to be an important variable influencing the decision to be tested: Gray et $a^{23}$ in a study investigating women's attitudes to online BRCA testing found that women who had been exposed to risk information had less positive beliefs about online BRCA testing, the most negative beliefs being held by those who had received risk information from experts. Kaphingst et $a l^{19}$ found that although participants were positive about the information on PGT provided on the study website, 49\% decided not to be tested, and the more information was viewed, the easier the decision. Wilde et $a l^{26}$ found that in patients before receiving information on DTC testing, $49 \%$ expressed interest; this percentage dropped to $40 \%$ after receiving detailed information. One study found that perception of a health condition as severe reduced the likelihood of presenting for testing. ${ }^{20}$

There appear to be conflicting opinions regarding the role of age in the decision to undergo testing, or to express concerns. Bloss et al ${ }^{16}$ suggest that younger people are more unsure about testing; Cherkas et $a l^{22}$ argue that younger people are not only significantly more likely to be aware, but also to express more interest in testing. Other studies, for example, Sweeny and $\operatorname{Legg}^{32}$ found no effect for gender or age apart from the fact that women perceived greater barriers and anticipated regret over testing. It has been suggested that non-white individuals are less likely to purchase and undergo DTC tests. ${ }^{16}$

\section{Consequences of having a DTC test}

One study, Bloss et al ${ }^{17}$ focused on the psychological, behavioural and clinical effects of having a DTC genome scan, and found that there was no effect on anxiety levels, dietary or exercise habits following the provision of risk score. There was, however, increased intention to undergo further clinical screening; this did not, however, translate into actual increase in screening. Higher risk scores predicted greater intention to pursue further screening. Test-related distress was associated with level of risk score, but over $90 \%$ of participants had no test-related distress. It is interesting to note in this study that $36 \%$ of participants shared their results with either a counsellor or a physician, and sharing of results with a physician was associated with lower fat intake and increased exercise. Gollust et al ${ }^{18}$ found a higher percentage (over 90\%) who stated that they would share their results with their physician, and $65 \%$ wanted health advice based on the results. There was some doubt expressed about the ability of their doctor to interpret DTC results. The majority (19 out of 23) of participants in the 'early users' study by McGowan et al ${ }^{28}$ stated that the results did not influence their health decisions or preventive behaviour, although some said that it might in the future. Participants in this study expressed concern about the lack of physician knowledge. McGuire et $a l^{24}$ found that $40 \%$ of those willing to undergo DTC testing would use the information without having to consult a physician, however, $53 \%$ of those who actually had DTC testing did discuss the results with their physician. These authors also demonstrated that those who considered the test result as (wrongly) diagnostic were significantly more likely to consult a physician post-test, and the majority thought that physicians had an obligation to help interpret the results. ${ }^{24}$

\section{DISCUSSION}

The primary aim of this systematic review was to collate evidence on the use of, and attitudes of the public to direct-to-consumer genomic tests. It is worth noting that only two papers report on participants who have actually used DTC testing, ${ }^{28,29}$ and that the recruitment and sampling methods in three large-scale studies ${ }^{16-20}$ included sampling frames that were not representative of the population at large, and indeed comprised individuals likely to have a knowledge of health and genetics greater than the average member of the public. This was also a weakness of a recent survey by Nature (in which $82.9 \%$ of respondents were scientists) that reported $18.2 \%$ of respondents had undergone genome analysis. ${ }^{33}$ Finally, despite claiming to investigate knowledge and attitudes to DTC testing, some research teams did so in a context which more closely resembled that of a genetic test requested with the support of a health-care professional.

Cherkas et al' acknowledged that their findings might 'diminish in an actual testing scenario' ( $\mathrm{p} 4$ ). In a study in which women's beliefs and intentions regarding DTC testing for BRCA were shown to be influenced by exposure to risk information, there is no discussion about how these findings might translate into actual testing. ${ }^{23}$ Two other papers contain acknowledgements that findings are limited by the hypothetical nature of the study. ${ }^{24,32}$ As the latter authors state, research on actual uptake of DTC tests is not easy to undertake. ${ }^{32}$ One reason for this is the problem of identifying and recruiting participants. This was overcome in an innovative way by McGowan et a ${ }^{28}$ who searched the Google search engine for individuals who had written blogs on DTC websites.

It is difficult to estimate uptake from data on intention to test; for example, Sanderson et $a^{34}$ found that although $63 \%$ of participants said they definitely would take the genetic test in question (for susceptibility to lung cancer), uptake was $38 \%$. A survey of people at risk of Huntington disease revealed that compared with an expected uptake of up to $80 \%$, only 5 and $20 \%$ were tested between 1988 and 1997.35

Various models are used to explain how people make health decisions, for example, the Health Belief Model, ${ }^{36}$ which has four main constructs that can explain behaviour - perceived seriousness, perceived susceptibility, perceived benefits and perceived barriers. In relation to this, several studies identified the attitudes of potential users of DTC tests, including concerns, perceived benefits and barriers. ${ }^{16,19,20,24,27,28}$ However, for reasons mentioned above, one should be cautious when using these findings to predict actual uptake of these tests, although uptake of targeted tests (eg, for risk of coronary heart disease) might be more popular with consumers.

For a person to give valid consent, there are three criteria that need to be fulfilled: the provision of appropriate information, the capacity to process that information and the voluntary nature of the consent. There is a suggestion that information provided on DTC websites may not represent a balanced picture in terms of risks and benefits. ${ }^{37}$ It is possible on a DTC website to order a PGT kit without giving consent (see 23andMe). ${ }^{38} \mathrm{~A}$ consent form is included with the kit, but this form relates only to the 23andWe scientific study and not the testing process itself. This means that consumers may still agree to the test without access to appropriate information, and possibly without full understanding of the consequences of the test. It is also possible that companies would consider return of a sample as sufficient evidence of consent.

Without receiving all the relevant information and giving valid consent, it is possible that consumers will embark on a DTC test without understanding the nature and implications of such a test.

Personal genome scans have only been available direct-to-consumers in the last decade. Despite the fact that company websites offering DTC tests state that these tests are for predicting the risk of developing any of a range of complex diseases (with some adding disclaimers that the tests are not for medical purposes), some participants thought that the test provided a diagnosis. In contrast, some early users were realistic about the nature of the tests undertaken and accepted their limitations. ${ }^{28}$ However, with 23andMe now including 
BRCA mutation testing of clinically relevant SNPs and carrier testing for conditions such as cystic fibrosis, positive results from these tests may have significant health or reproductive implications. Unless people understand that the majority of DTC test results represent a percentage risk or probability as opposed to a diagnosis, their use is to a certain extent limited. Without studies of actual users of DTC tests, it is difficult to predict how people would react to the results. However, most study samples in this review consisted of people with specific knowledge of health and/or technology. It should be noted, however, that although some participants in these large studies ${ }^{17,18}$ did have genomic tests performed, it may not be appropriate to consider them in the same way as commercial DTC tests, as they were taken in the context of a study, with the accompanying support and information.

As well as possible behavioural changes, there may well be an effect on both the way people consult with their physicians, and the effect of onward referrals as a result of consultations following a DTC test. There is evidence from the studies in this review that intending to, or actually having a DTC test was associated with increased consultation (or planned consultation) with health professionals. There are two concerns for consideration here: the first is the level of specialist genetic knowledge in primary care (concerns were expressed in two studies), ${ }^{18,28}$ the second is the possible increased cost of health care because of increased demand for consultation and related screening or diagnostic tests.

There is evidence that a large percentage of the population use the Internet to access health information. ${ }^{39}$ A possible outcome of this is that individuals may, as consumers rather than patients, access DTC tests that may not have proven clinical validity or utility. In contrast, individuals consulting a health professional for information on such tests would benefit from the health professional's ability to access information on the clinical validity and utility of these tests. ${ }^{40}$ Consumers of DTC tests may be exposed to information and advertising material that is not fully informative and some tests based on weak associations. They may therefore be purchasing tests that are of little value related to their health-care management. However, in cases where the testing indicates that an individual is a carrier for a recessive condition or has inherited a mutation that confers significant additional risk (such as a BRCA1 mutation) to the individual and potentially their biological relatives, genetic counselling by a qualified professional will usually be necessary to ensure the information is able to be used by the individual and their family members in a way that promotes their wellbeing.

\section{CONCLUSIONS}

It is likely that there will be public interest in DTC testing, assuming that awareness increases and this interest will have educational implications, both for certain sectors of the general population and for health-care professionals. Factors such as age, professional status, marital status and health information seeking behaviour have been cited as predictors of not only awareness, but level of knowledge and perception of risks. For this reason, more research is needed to identify the experiences of actual users of DTC tests. Unfortunately, there may be few users and they may not be easy to reach if they are motivated to guard their privacy by bypassing a health-care professional, to avoid information being incorporated into their medical record. Companies may also be keen to safeguard their own clientele. ${ }^{32}$

Finally, a public that is more aware and knowledgeable will need to be well informed about issues such as privacy, the quality of DTC tests and the evidence that supports them. However, those who wish to consult a health-care professional following a DTC test for evidence-based advice on their future health care and lifestyle will inevitably place additional pressures on the health service.

\section{CONFLICT OF INTEREST}

The authors declare no conflict of interest.

\section{ACKNOWLEDGEMENTS}

This study was undertaken as part of the work of Unit 2, Work Package 7 of the EuroGentest2 Coordination Action 2011 project. The project is funded by the European Commission, EU Contract no.: HEALTH-F4-2010-261469. We thank Professor Helena Kaariainen for her helpful comments on the text.

1 Burton H: Genetics and Mainstream Medicine. Cambridge, UK: Public Health Genetics Foundation, 2011.

2 McBride CM, Wade C, Kaphingst KA: Consumers' views of direct-to-consumer genetic information. Annu Rev Genomics Hum Genet 2010; 11: 427-446.

3 National Human Genome Research Institute: A Catalog of Published Genome-Wide Association Studies. National Human Genome Research Institute, 2011 (Online). Available at http://www.genome.gov/26525384 (accessed 8 September 2011).

4 McGuire AL, Burke W: An unwelcome side effect of direct-to-consumer personal genome testing. Raiding the medical commons. J Am Med Assoc 2008; 300: 2669-2671.

$5 \mathrm{Ng}$ PC, Murray SS, Levy S, Venter JC: An agenda for personalized medicine. Nature 2009; 461: 724-726.

6 Godard B, Kaariainen H, Kristoffersson U, Tranebjaerg L, Coviello D, Ayme S: Provision of genetic services in Europe: current practices and issues. Eur J Hum Genet 2003; 11 (Suppl 2): S13-S48.

7 US Food and Drug Administration: Letter to Pathway Genomics Corporation Concerning the Pathway Genomics Genetic Health Report 2010 (Online). Available at http://www.fda.gov/MedicalDevices/ResourcesforYou/Industry/ucm211866.htm (accessed 21 July 2011).

8 Lewis C, Kent A, Skirton H, Coviello D: EuroGentest patient information leaflets: a free resource available in over 20 languages. Eur J Hum Genet 2009; 17: 732-732.

9 Messner DA: Informed choice in direct-to-consumer genetic testing for Alzheimer and other diseases: lessons from two cases. New Genet Soc 2011; 30: 59-72.

10 van El CG, Cornel MC: Genetic testing and common disorders in a public health framework. Eur J Hum Genet 2011; 19: 377-381.

11 Borry P: Statement of the ESHG on direct-to-consumer genetic testing for healthrelated purposes. Eur J Hum Genet 2010; 18: 1271-1273.

12 Victor L: Systematic reviewing. Social Res Update 2008.

13 Centre for Reviews and Dissemination: Systematic Reviews. York: CRD, University of York, 2008.

14 Kmet LM, Lee RC, Cook LS: Standard Quality Assessment Criteria for Evaluating Primary Research Papers from a Variety of Fields. Alberta Heritage Foundation for Medical Research, 2004 (Online), Edmonton, Canada. Available at http://www.ine.ca/ documents/HTA-FR13.pdf (accessed 1 February 2012).

15 Braun V, Clarke V: Using thematic analysis in psychology. Qualitative Res Psychol 2006; 3: 77-101.

16 Bloss CS, Ornowski L, Silver E et al: Consumer perceptions of direct-to-consumer personalized genomic risk assessments. Genet Med 2010; 12: 556-566.

17 Bloss CS, Schork NJ, Topol EJ: Effect of direct-to-consumer genomewide profiling to assess disease risk. N Engl J Med 2011; 364: 524-534.

18 Gollust SE, Gordon ES, Zayac C et al: Motivations and perceptions of early adopters of personalized genomics: perspectives from research participants. Public Health Genomics 2011.

19 Kaphingst KA, McBride CM, Wade C, Alford SH, Brody LC, Baxevanis AD: Consumers' use of web-based information and their decisions about multiplex genetic susceptibility testing. J Med Internet Res 2010; 12: e41.

20 McBride CM, Hensley-Alford S, Reid RJ, Larson EB, Baxevanis AD, Brody LC: Characteristics of users of online personalized genomic risk assessments: implications for physician-patient interactions. Genet Med 2009; 11: 582-587.

21 O'Neill SC, White DB, Sanderson SC et al: The feasibility of online genetic testing for lung cancer susceptibility: uptake of a web-based protocol and decision outcomes. Genet Med 2008; 10: 121-130.

22 Cherkas LF, Harris JM, Levinson E, Spector TD, Prainsack B: A survey of UK public interest in internet-based personal genome testing. PLOS ONE 2010; 5: E13473.

23 Gray SW, O'Grady C, Karp L et al: Risk information exposure and direct-to-consumer genetic testing for BRCA mutations among women with a personal or family history of breast or ovarian cancer. Cancer Epidemiol Biomark Prevent 2009; 18: 1303-1311.

24 McGuire AL, Diaz CM, Wang T, Hilsenbeck SG: Social networkers' attitudes toward direct-to-consumer personal genome testing. Am J Bioethics 2009; 9: 3-10.

25 Perez GK, Cruess DG, Cruess S et al: Attitudes toward direct-to-consumer advertisements and online genetic testing among high-risk women participating in a hereditary cancer clinic. J Health Commun 2011; 16: 607-628. 
26 Wilde A, Meiser B, Mitchell PB, Hadzi-Pavlovic D, Schofield PR: Community interest in predictive genetic testing for susceptibility to major depressive disorder in a large national sample. Psychol Med 2011; 41: 1605-1614.

27 Wilde A, Meiser B, Mitchell PB, Schofield PR: Public interest in predictive genetic testing, including direct-to-consumer testing, for susceptibility to major depression: preliminary findings. Eur J Hum Genet 2010; 18: 47-51.

28 McGowan ML, Fishman JR, Lambrix MA: Personal genomics and individual identities: motivations and moral imperatives of early users. New Genet Soc 2010; 29: 261-290.

29 Su Y, Howard HC, Borry P: Users' motivations to purchase direct-to-consumer genomewide testing: an exploratory study of personal stories. J Commun Genet 2011; 2: 135-146.

30 Ortiz AP, Lopez M, Flores LT et al: Awareness of direct-to-consumer genetic tests and use of genetic tests among Puerto Rican adults, 2009. Prevent Chronic Disease 2011; 8: A110.

31 Leighton JW, Valverde K, Bernhardt B: The general public's understanding and perception of direct-to-consumer genetic test results. Public Health Genomics 2012; 15: $11-21$.

32 Sweeny K, Legg AM: Predictors of interest in direct-to-consumer genetic testing. Psychol Health 2011; 26: 1259-1272.
33 Maher B: Nature readers flirt with personal genomics. Nature 2011; 478: 19.

34 Sanderson SC, O'Neill SC, Bastian L, Bepler G, McBride CM: What can interest tell us about uptake of genetic testing? intention and behavior amongst smokers related to patients with lung cancer. Public Health Genomics 2010; 13: 116-124.

35 Harper PS: Ten years of presymptomatic testing for Huntington's disease: the experience of the UK Huntington's Disease Prediction Consortium. J Med Genet 2000; 37: 567-571.

36 Stretcher V, Rosenstock IM: The health belief model; in Glanz K, Lewis FM, Rimer BK (eds): Health Behavior and Health Education: Theory, Research and Practice. San Francisco: Jossey-Bass, 1997.

37 Vashlishan Murray AB, Carson MJ, Morris CA, Beckwick J: Illusions of scientific legitimacy: misrepresented science in the direct-to-consumer genetic-testing marketplace. Trends Genet 2010; 26: 459-461.

38 23andMe. 2011, (Online). Available at https://www.23andme.com (accessed 22 September 2011).

39 Hesse BW, Nelson DE, Kreps GL et al: The impact of the internet and its implications for health care providers: findings from the first Health Information National Trends Survey. Arch Intern Med 2005; 165: 2618-2624.

40 Draper J: Whose welfare in the labour room? A discussion of the increasing trend of fathers' birth attendance. Midwifery 1997; 13: 132-138.

Supplementary Information accompanies the paper on European Journal of Human Genetics website (http://www.nature.com/ejhg) 